\section{Infection 10 months after insertion of Nexplanon implant}

In 2013, four cases of infection after insertion of a Nexplanon implant were reported in this journal. ${ }^{12}$ In the first case symptoms began a few weeks after insertion. ${ }^{1}$ In the next three cases symptoms occurred 7-10 days later, all patients having atopic eczema. ${ }^{2}$ I report a case of infection 10 months after insertion of an implant in a patient with atopic eczema.

A 28-year-old woman presented with a 3-day history of redness, discomfort, and swelling over the subdermal site of an implant that I had fitted 10 months earlier. She had had no other problems since then. She did not have a history of trauma or broken skin adjacent to the area, but she did have a history of atopic eczema, which had been well controlled at the time of insertion.

On examination she was comparatively well with a mild fever of $37.7^{\circ} \mathrm{C}$. The inflammation, swelling, and

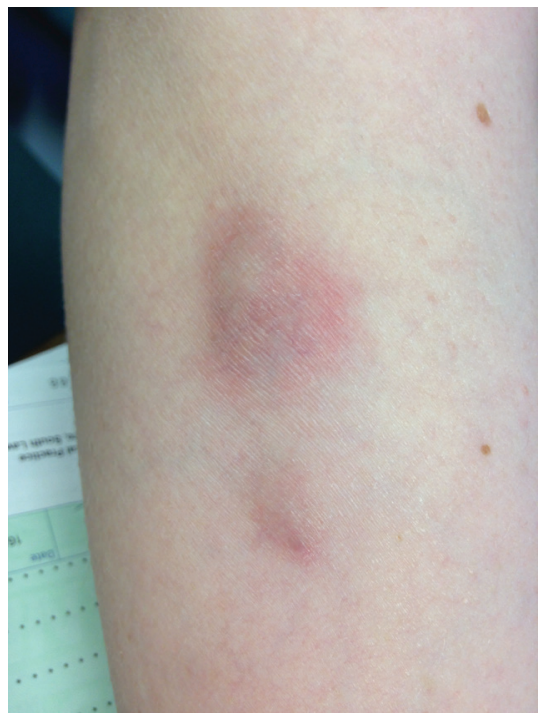

Figure 1 Inflammation and swelling over site of implant.

warmth over the site of her implant were greater towards the proximal end (figure 1), which was fluctuant on palpation.

I advised her that she had an infection and recommended that the implant be removed. I did so that morning using the standard technique. A thin purulent discharge was extruded. I sent a swab and the implant for microbiological analysis, prescribing the patient a course of flucloxacillin.

Cultures showed growth of Staphylococcus aureus sensitive to flucloxacillin from both the discharge and the implant. On review a week later the wound had healed.

The local microbiology department thought that the infection was most likely to be a blood-borne bacteraemia that had seeded the implant. Infection was unlikely to have been introduced at the time of insertion because of the timeline of events. Patients with eczema carry a significantly higher load of $S$. aureus, thereby increasing the chance of immediate and delayed infection after insertion of an implant.

The patient was keen to have a further implant despite her infection and comprehensive discussion about other methods of contraception. We agreed to optimise her eczema treatment for a month and to fit the implant into her other arm. Six months later she had no further problems, having been advised about the ongoing management of her eczema.

Patients with atopic eczema and other generalised dermatological conditions should be carefully counselled about 
the risk of infection immediately and at any time after insertion of an implant. Their condition should be optimally treated before and after insertion.

\section{Jonathan Richold}

Correspondence to Dr Jonathan Richold, South Lawn Medical Practice, Heavitree Health Centre, Exeter EX1 2RX, UK; jonathan.richold@nhs.net

Competing interests None declared.

Patient consent Obtained.

Provenance and peer review Not commissioned; internally peer reviewed. (C) Article author(s) (or their employer(s) unless otherwise stated in the text of the article) 2018. All rights reserved. No commercial use is permitted unless otherwise expressly granted.

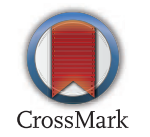

BMJ Sex Reprod Health 2018;44:70-71.

doi:10.1136/bmjsrh-2017-101885

\section{REFERENCES}

1 Chaudhry F. Adverse reaction to Nexplanon. J Fam Plann Reprod Health Care 2013;39:231-2.

2 Partridge R, Bush J. Infections postNexplanon insertion. J Fam Plann Reprod Health Care 2013;39:309-10. 